\section{Label-free in vivo optical imaging of microvasculature and oxygenation level}

\author{
Dan Fu, ${ }^{a}$ Thomas E. Matthews, ${ }^{\text {b }}$ Tong Ye, ${ }^{\text {c }}$ Ivan R. Piletic, ${ }^{b}$ \\ and Warren S. Warren ${ }^{\mathrm{b}, *}$ \\ aPrinceton University, Department of Chemistry, Princeton, \\ New Jersey 08544 \\ ${ }^{\mathrm{b}}$ Duke University, Department of Chemistry, Durham, North \\ Carolina 27708 \\ 'University of Alabama at Birmingham, Department of \\ Neurobiology, Birmingham, Alabama 35294
}

\begin{abstract}
The ability to perform high-resolution imaging of microvasculature and its oxygenation is very important in studying early tumor development. Toward this goal, we improved upon our excited state absorption (ESA)based imaging technique to allow us to not only image hemoglobin directly but also differentiate between oxyand deoxyhemoglobin in tissue. We demonstrate the separation of arterioles from venules in a live nude mouse ear using our imaging technique. ๑ 2008 Society of Photo-Optical Instrumentation Engineers. [DOI: 10.1117/1.2968260]
\end{abstract}

Keywords: two-photon absorption; excited state absorption (ESA); multiphoton microscopy; microvasculature imaging; oxygenation imaging.

Paper 08107LR received Mar. 28, 2008; revised manuscript received Jun. 9, 2008; accepted for publication Jun. 12, 2008; published online Aug. 26, 2008.

Blood vessel structure and oxygenation provides important information about angiogenesis and hypoxia in tumor development. ${ }^{1-3}$ Microscopic imaging provides a very intuitive way of visualizing the angiogenic vasculature differences between tumors and healthy tissue. Recent developments in confocal and multiphoton fluorescence microscopy (MFM) based on fluorescent labeling of blood plasma or vessel walls allow submicrometer-resolution imaging of microvasculature and provide sectioning capabilities. ${ }^{4-6}$ These methods can measure a number of important parameters such as blood flow rate, blood vessel diameter, vascular density, and endothelial permeability. However, the necessary use of a tracer or fluorescence tag is an obvious limitation, especially for human studies. Another important aspect that is missing from confocal or multiphoton imaging of blood vessels is the oxygenation level, which is a key determinant of the physiological state of tissue. Because of the irregular pattern and organization of vasculature in tumors, some cancerous cells are located more than $100 \mu \mathrm{m}$ (the diffusion limit for oxygen) away from blood vessels and become hypoxic. Tumor hypoxia has been suggested as a cause of malignant transformation and a source of resistance to current cancer therapies. Therefore, measuring blood oxygen delivery and hypoxia at

*Address all correspondence to Warren S. Warren, Duke Univ., Dept. of Chemistry, 124 Science Dr., Durham, NC; Tel: 919-660-1506; Fax: 919-287-2459; E-mail: warren.warren@duke.edu. the microvascular level could be of important prognostic value for tumor diagnosis and treatment. ${ }^{7}$

We have developed a new excited state absorption (ESA)based method to image hemoglobin and melanin in tissue without any labeling. ${ }^{8,9}$ Previously, we have demonstrated high-resolution in vivo imaging of the blood vessels in mouse ears. $^{8}$ In this report, we improved the sensitivity by shifting the pump and probe pulses toward longer wavelengths (720 to $760 \mathrm{~nm}$ and $810 \mathrm{~nm}$ ), which will also give us deeper penetration in tissue. More importantly, the ratios of two-color ESA signal sizes of oxyhemoglobin and deoxyhemoglobin are very different when we simply switch the pump and probe sequence. Therefore, it is possible to determine their ratio by comparing images taken under these two different conditions. We are able to use this technique to differentiate between arterioles and venules in a live mouse ear. Our two-color ESA imaging technique could potentially be very useful in studying the microvasculature and oxygenation level changes in early tumor development.

The experimental setup has been described in detail previously. ${ }^{9,10}$ Here, we made some modifications to the original setup. Both the $810-\mathrm{nm}$ beam and the $740-\mathrm{nm}$ beam were modulated independently with two acousto-optic modulators (AOMs). We can choose to use either beam as the pump by simply activating the AOM in the desired beam path. The pump and probe beams were then combined on a dichroic mirror (Chroma, DCXR760) and sent either to a home-built upright microscope $(10 \times$ objective, $\mathrm{NA}=0.25$, infinity corrected) for solution measurements or a home-built laser scanning microscope (Olympus PlanApo $20 \times$ objective with $\mathrm{NA}=0.70$ ) for mouse ear imaging. The mouse was placed on a custom-designed holder attached to a motorized XYZ stage to allow easy access to the blood vessels.

We first studied $0.75 \mathrm{mM}$ oxy- and deoxyhemoglobin solutions and compared the results to a $30 \mathrm{mM}$ Rhodamine $6 \mathrm{G}$ (R6G) solution, which has two-photon absorption (TPA) but no linear absorption at these wavelengths. From Fig. 1(a), we can obtain a cross-correlation length of 329 fs FWHM, much larger than that calculated for transform-limited pulses. This is mainly due to pulse stretching from the two AOMs, which could be readily compensated with a prism pair if necessary. Figures 1(b) and 1(c) show the ESA signals of oxyhemoglobin and deoxyhemoglobin under different experimental conditions. Essentially what we are measuring is the difference in probe transmission between pump on and pump off, and the resulting signal can be expressed as:

$$
\Delta \mathbf{I}_{2}=-\int N_{0} \sigma\left(\omega_{1}\right)\left[\sigma^{\prime}\left(\omega_{2}\right)-\sigma\left(\omega_{2}\right)\right] \mathbf{I}_{1} \mathbf{I}_{2} / \hbar \omega_{1} \exp (-\Delta t / \tau) \mathrm{d} z
$$

where $N_{0}$ is the molecular concentration; $\sigma$ and $\sigma^{\prime}$ are the linear absorption cross sections of the ground state and excited states, respectively; $\omega_{1}$ and $\omega_{2}$ represent the pump and probe frequencies, respectively; and $\tau$ is the lifetime of the excited state. Equation (1) implies that we will see exponential decay when the pump probe delay $\Delta t$ is varied over positive values. The signal will be zero for negative delays (when the probe is before the pump). This is because in the absence of a pump pulse, there is no excited state population from which to give rise to probe transmission changes. Both oxy- 


\section{JBO LETTERS}
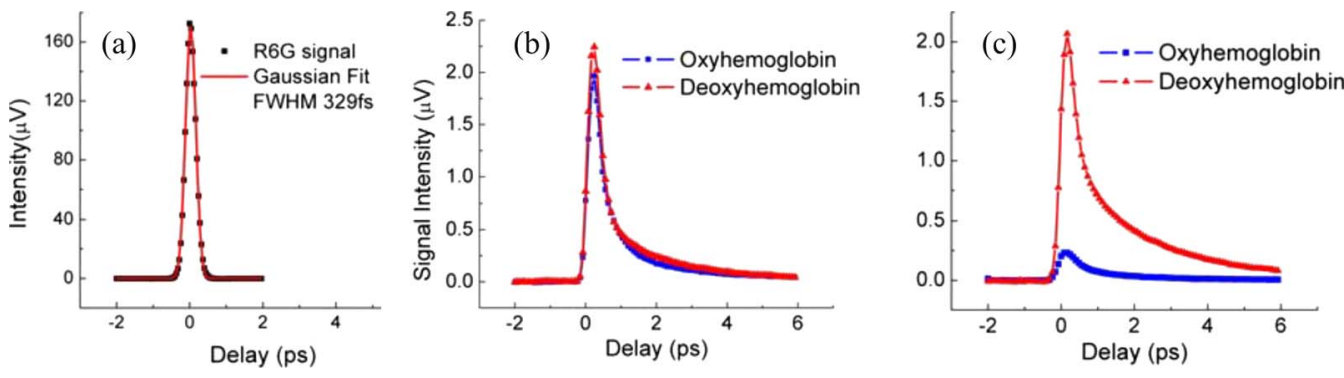

Fig. 1 (a) TPA signal from R6G with 810-nm pump at $10 \mathrm{~mW}$ and 740-nm probe at $6.4 \mathrm{~mW}$. (b) ESA signal from oxyhemoglobin and deoxyhemoglobin with pump wavelength at $810 \mathrm{~nm}$ and probe wavelength at $740 \mathrm{~nm}$; pump power was $10 \mathrm{~mW}$ and probe power was $6.4 \mathrm{~mW}$. (c) ESA signal from oxyhemoglobin and deoxyhemoglobin with pump wavelength at $740 \mathrm{~nm}$ and probe wavelength at $810 \mathrm{~nm}$; pump power was $2.4 \mathrm{~mW}$ and probe power was $10 \mathrm{~mW}$.

hemoglobin and deoxyhemoglobin exhibited long signal decays, which can be fitted with a double exponential decay with short time constant of around 200 fs and long time constant of 2 ps, with no significant difference between the two. We can obtain an equivalent TPA cross section from Eq. (1):

$$
\sigma^{(2)}\left(\omega_{1}, \omega_{2}\right)=\sigma\left(\omega_{1}\right)\left[\sigma^{\prime}\left(\omega_{2}\right)-\sigma\left(\omega_{2}\right)\right] \exp (-\Delta t / \tau) / 2 \hbar \omega_{1} .
$$

The equivalent TPA cross section depends on both pump and probe frequency and can be estimated by comparison ${ }^{11}$ with the known R6G TPA cross section at $800 \mathrm{~nm}$. For the 810-nm pump case, oxyhemoglobin and deoxyhemoglobin have comparable signal sizes with an estimated TPA cross section of $7.8 \mathrm{GM}$, while for the 740-nm pump case, the deoxyhemoglobin signal is nearly 10 times as strong as the oxyhemoglobin signal. The stronger linear absorption of deoxyhemoglobin at $740 \mathrm{~nm}$ is directly translated into stronger ESA signal according to Eq. (1). Of course, other factors such as excited state absorption cross section and competition from bleaching could also contribute. This result is very important because we can simply take two different pump-probe measurements and be able to separate oxyhemoglobin from deoxyhemoglobin or even quantify their ratio.

The nude mouse ear is a very good model to demonstrate our microvasculature and oxygenation level imaging capabilities without surgical intervention. We first imaged the blood vessels inside an anesthetized nude mouse ear with the $810-\mathrm{nm}$ beam as the pump and the $735-\mathrm{nm}$ beam as the probe. (The probe wavelength was chosen to give the highest power and best stability of the laser.) Figure 2 shows the bright-field image of an area rich with microvessels and the corresponding two-color ESA laser scanning images at increasing penetration depth. The pump and probe interpulse delay was set at $500 \mathrm{fs}$ to avoid cross-phased modulation (XPM) artifacts or TPA interference from other molecular or structural components (some features on the surface can still be observed), which happens only when the pump and probe pulse overlap in time. The size for these images is $512 \mu \mathrm{m}$ $\times 512 \mu \mathrm{m}$ with $256 \times 256$ pixels. Frame acquisition time is $52 \mathrm{~s}$, which is currently limited by the time constant of the lock-in amplifier. The twisting 3-D capillaries with diameters in the range of 5 to $10 \mu \mathrm{m}$ can be easily identified. We can also image the blood vessels with the $735-\mathrm{nm}$ beam as the pump and the $810-\mathrm{nm}$ beam as the probe. Figure 3 shows the bright-field image of another mouse ear with dense capillaries and the corresponding two-color ESA laser scanning images at increasing penetration depths. Higher SNR was obtained compared to 810-nm pumped laser scanning images. One reason for this could be that the transmission of 735-nm light is much lower than 810-nm light because of scattering and therefore leads to lower light collection on the detector. ${ }^{8}$ It could also be caused by the change of mode mismatching when the pump probe sequence is switched.

The most important aspect of our improved imaging method is differentiating arterioles from venules. In Fig. 4, we compare the acquired laser scanning images at the same depth $(30 \mu \mathrm{m})$ with different pump-probe conditions side to side. We can easily see that these two images provide very similar location and size information for the blood vessels but have different contrasts. Although it is hard to directly obtain a ratio image because of frame shifts between two images as well as small SNR, we are still able to differentiate arteriole from venule with semiquantitative analysis using results from solution measurements. The blue arrows indicate the vessels that have much less signal compared to vessels highlighted with red arrows when imaged with the 810-nm pump, which tells us that they are venules, and the red arrows are pointed at arterioles (color online only). We can even analyze the capillaries in the same way. However, other factors such as scattering loss and blood circulation might also contribute to the
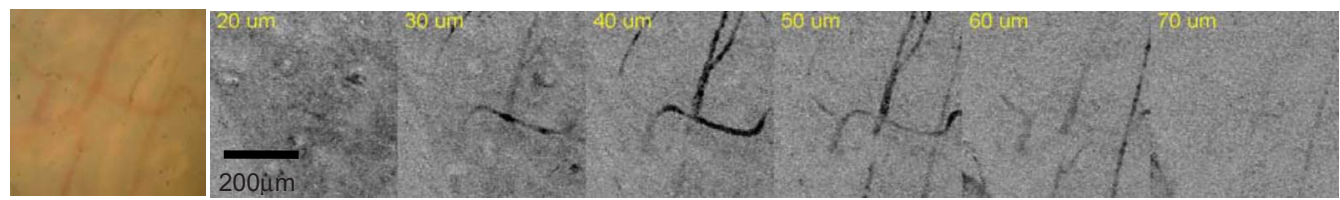

Fig. 2 Bright-field image and corresponding two-color ESA images at increasing imaging depth (pump at $810 \mathrm{~nm}$ with 3-mW power and probe at $735 \mathrm{~nm}$ with 2-mW power). (Color online only.) 


\section{JBO LETTERS}

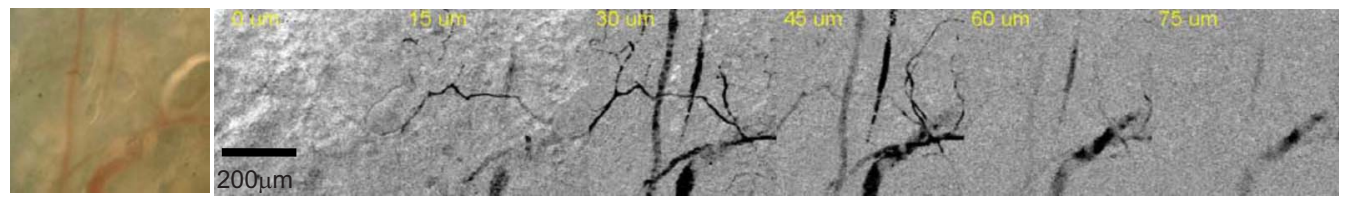

Fig. 3 Bright-field image and corresponding two-color ESA images at increasing imaging depth (pump at $735 \mathrm{~nm}$ with 4 -mW power and probe at $810 \mathrm{~nm}$ with 1.6-mW power). (Color online only.)

ratio change, and therefore a careful calibration is necessary before we do any quantitative analysis with only two images.

This paper demonstrates the feasibility of utilizing a twocolor ESA microscope to image microvasculature and its oxygenation in vivo. Individual blood vessels with $<5$ to $10 \mu \mathrm{m}$ diameter can be easily identified. More importantly, we are able to discern arterioles and venules with two sequential images by switching the pump and probe wavelengths. To further improve the SNR, more than five times the optical power (up to $30 \mathrm{~mW}$ ) can be used for deep tissue imaging (up to $300 \mu \mathrm{m})$. New wavelength combinations may also dramatically increase the equivalent TPA cross section and therefore increase the SNR. We should also be able to obtain two-times higher SNR with precompensation of dispersion to significantly shorten the pulse durations after the objective. Another important aspect is spatial beam mode matching for the pump and probe. Currently, the pump and probe beam come from different sources and pass through many different optical elements, and therefore the mode matching both spatially and temporally is not perfect. We should be able to have much better mode matching with two pulses coming from the same
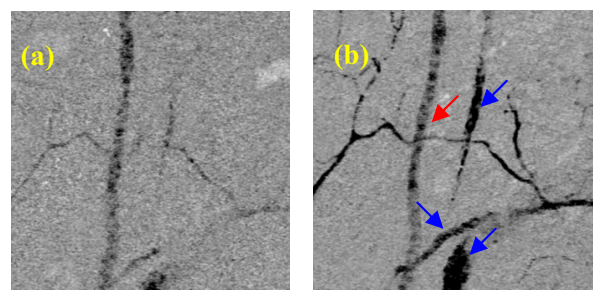

Fig. 4 Comparison of the laser scanning images of blood vessels with different pump-probe combinations: (a) pump at $810 \mathrm{~nm}$ with 2.7-mW power and probe at $735 \mathrm{~nm}$ with $2.6-\mathrm{mW}$ power, and (b) pump at $735 \mathrm{~nm}$ with $4-\mathrm{mW}$ power and probe at $810 \mathrm{~nm}$ with 1.6-mW power. broadband source with wavelength multiplexing. With better SNR and a simplified experimental setup, both imaging speed and penetration depth can be increased significantly. Epimode imaging is also possible and is discussed in another paper. ${ }^{10}$ Our ESA imaging technique could prove to be an invaluable diagnostic tool in noninvasive imaging of tumor angiogenesis and hypoxia.

\section{References}

1. D. M. McDonald and P. L. Choyke, "Imaging of angiogenesis: from microscope to clinic," Nat. Med. 9, 713-725 (2003).

2. P. C. Lin, "Optical imaging and tumor angiogenesis," J. Cell. Biochem. 90, 484-491 (2003).

3. B. S. Sorg, B. J. Moeller, O. Donovan, Y. T. Cao, and M. W. Dewhirst, "Hyperspectral imaging of hemoglobin saturation in tumor microvasculature and tumor hypoxia development," J. Biomed. Opt. 10, 044004 (2005)

4. E. B. Brown, R. B. Campbell, Y. Tsuzuki, L. Xu, P. Carmeliet, D. Fukumura, and R. K. Jain, "In vivo measurement of gene expression, angiogenesis, and physiological function in tumors using multiphoton laser scanning microscopy," Nat. Med. 7, 864-868 (2001).

5. R. K. Jain, L. L. Munn, and D. Fukumura, "Dissecting tumour pathophysiology using intravital microscopy," Nat. Rev. Cancer 2, 266276 (2002).

6. D. Kleinfeld, P. P. Mitra, F. Helmchen, and W. Denk, "Fluctuations and stimulus-induced changes in blood flow observed in individual capillaries in layers 2 through 4 of rat neocortex," Proc. Natl. Acad. Sci. U.S.A. 95, 15741-15746 (1998).

7. S. Kizaka-Kondoh, M. Inoue, H. Harada, and M. Hiraoka, "Tumor hypoxia: a target for selective cancer therapy," Cancer Sci. 94, 10211028 (2003)

8. D. Fu, T. Ye, T. E. Matthews, B. J. Chen, G. Yurtserver, and W. S Warren, "High-resolution in vivo imaging of blood vessels without labeling," Opt. Lett. 32, 2641-2643 (2007).

9. D. Fu, T. Ye, T. E. Matthews, G. Yurtsever, and W. S. Warren, "Twocolor, two-photon, and excited-state absorption microscopy," $J$. Biomed. Opt. 12, 054004 (2007)

10. Y. Tong, F. Dan, E. M. Thomas, H. Lian, D. S. John, and S. W. Warren, "Two-photon absorption and transient photothermal imaging of pigments in tissues," Proc. SPIE 6842, 684206 (2008).

11. P. F. Tian and W. S. Warren, "Ultrafast measurement of two-photon absorption by loss modulation," Opt. Lett. 27, 1634-1636 (2002). 